\title{
Neural Correlates of Error Awareness
}

Tilmann A. Klein', Tanja Endrass' ${ }^{\prime}$ Norbert Kathmann', Jane Neumann ${ }^{\prime}$, D. Yves von Cramon ${ }^{l} \&$ Markus Ullsperger $^{l}$,

Max Planck Institute for Human Cognitive and Brain Sciences, Leipzig, Germany;

${ }^{2}$ Humboldt -University, Berlin, Germany

email:tklein@cbs.mpg.de

MAX $\mid$ FOR

PLANCK HUGAN COGITEE AND BRAIN SGIENCES

INSTITUTE LEPZZ

\section{Introduction}

- Role of conscious error perception for action outcome optimization

- Event related potentials (ERP; Nieuwenhuis et al,, 2001, Endrass et al, 2005): modulation of the error positivity (Pe) by error awareness - a normal Pe was found in case of an aware error, a diminished Pe was observed in case of an unaware error; the error related negativity (ERN) was present on aware and unaware errors

- Behavioural: post-error slowing (PES) only after an aware error

- Rostral cingulate zone (RCZ; most probable generator of the ERN) active on error processing (Debener et al., 2005)

Research questions:

1. Are there sizeable differences in RCZ activity or in the activity of other error processing related brain areas due to error awareness?

2. What are the behavioural correlates of error awareness?

\section{Methods}

1

a) Participants and Task

- Thirteen healthy right handed subjects ( 8 female, mean age $=26,15$ years)

- Antisaccade task (Nieuwenhuis et al., 2001; 476 Trials; see Figure 1) - Brief precue (to increase error rate) was presented at the position where was presented at the position where
the gaze should be directed to (to the gaze should be directed to (to
reduce predictability in $33 \%$ of reduce predictability in $33 \%$ of
the trials the precue was presented at the position of the following peripheral stimulus)

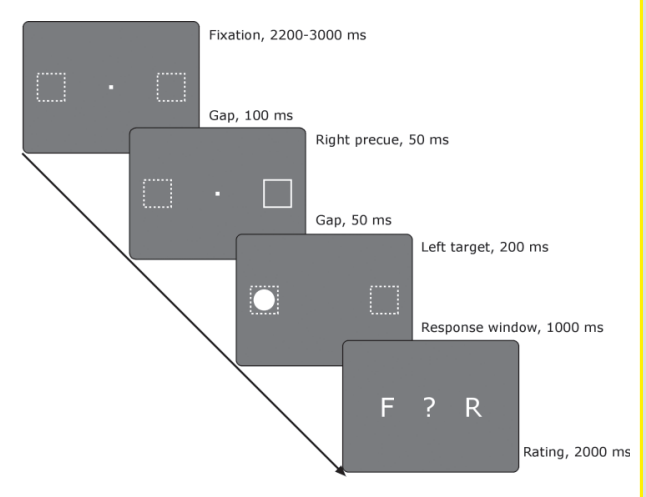
Figure 1. Schematic of the antisaccade task. Average trial du-
ration: $6 \mathrm{~s}$.

b) Data accquisition and Eyetracker

- Data was acquired with a standard fMRI protocol at $3 \mathrm{~T}$

- Data Processing: LIPSIA (Lohmann et al., 2001)

- $\mathrm{z}=2.33 ; \alpha=0.01$; volume $>81 \mathrm{~mm}^{3}$ (Monte Carlo Simulation: 0.05 corrected)

- View Point Eye tracker (infrared light based; Arrington Research)

- Temporal resolution: $60 \mathrm{~Hz}$; spatial resolution: app. $0.15^{\circ}$ visual arc

\section{2}

c) Region of Interest (ROI)

- Extended metaanalysis of 55 studies on error processing, response conflict, decision uncertainty and negative feedback (based on Rid derinkhof et al., 2004)

Analysis was based on Activation Likelihood Estimation (ALE;

Turkeltaub et al., 2002; see Figure 2 for results)

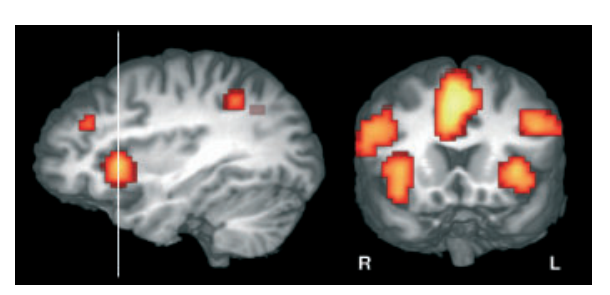

Figure 2. Results of the metaanalysi
$(\mathrm{x}=-32)$ and a coronal slice $(\mathrm{y}=15)$

\section{Results}

a) Behavioural

- Errors faster than correct responses (see Figure 3 to 6 for details)

- Considerable variance in PES across subjects; PES in 9 out of 13 subjects

- Lower error rate after an aware error

- False alarm rate: $3 \%$; rate of not classifiable trials due to technical problems: $6.1 \%$

3 Figure 3. Accuracy in trial $\mathrm{N}$

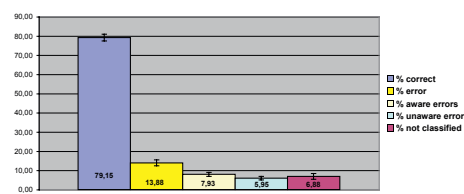

5
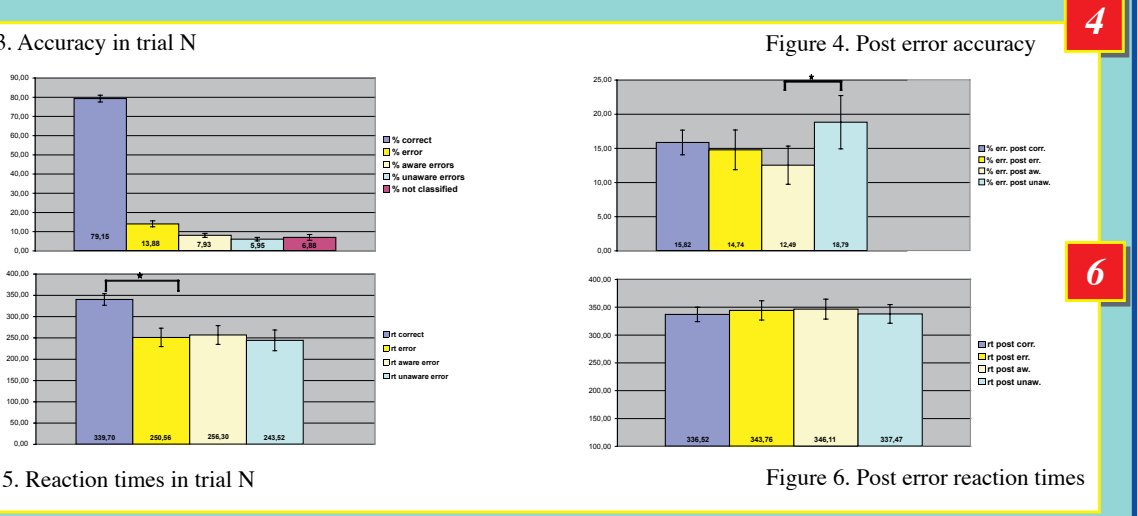

\section{Results}

b) FMRI

- Error > Correct: Pre-SMA, RCZ and Insula (see Table 1 \& Figure 7)

- Aware > Unaware: Left insula (see Table $1 \&$ Figure 7)

Timecourses: Greater activity in the insular cortex for aware errors, no such difference can be found in RCZ (see Figure 7)

- Parametric second-level analysis with post-error reaction time as a regressor: the more a person slowed down after an aware error, the more activity can be found in the pre-SMA ( $\mathrm{x}=$ $1, \mathrm{y}=6, \mathrm{z}=48$; see Figure 8)

Table 1

Table 1. Brain regions, Brodmann areas, and Talairach coordinates $(\mathrm{x}, \mathrm{y}, \mathrm{z})$ of voxels co-varying significantly wit error processing and error awareness.
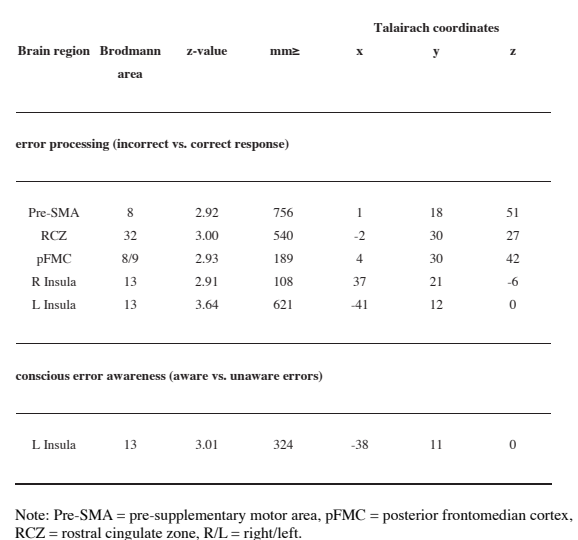

\section{7}

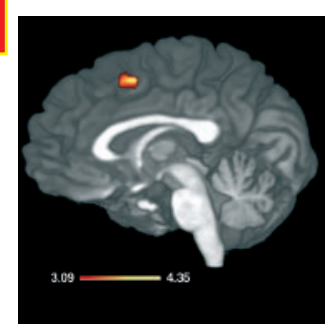

Figure 8. Results of the parametric second-level anlysis of brain activity rela-
ted to aware errors using post-error slowing as a r ficant correlation in the $\operatorname{pre-SMA}(x=1, y=6, z=$

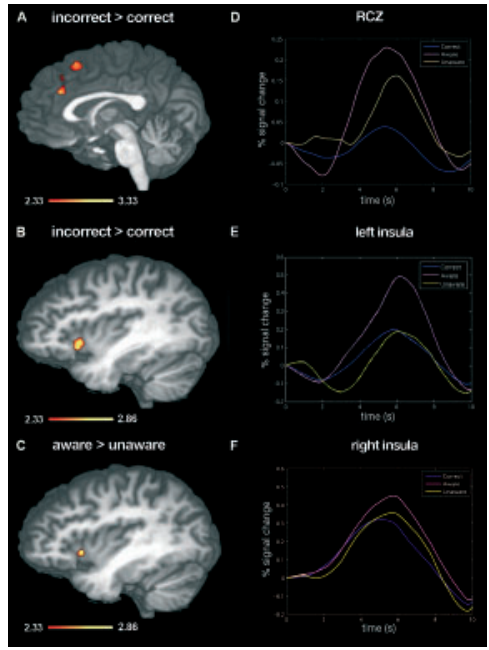

Figure 7. fMRI results. A and B: general errorrelated brain activity revealed by the contrast erro vs. correct response (sagittal slices at $\mathrm{x}=-2$ and -41 , error perception revealed by the contrast aware vs. (sagittal slice at $\mathrm{x}=-38$ ). D, E and $\mathrm{F}$ averaged time series of the BOLD response for
aware and unaware errors in the RCZ ( $; x=-2, y=$ $30, z=27$ ), the left anterior insula ( $E ; x=-38, y=$

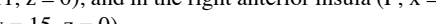

\section{Discussion}

$\mathrm{RCZ}$

- Active on both error types (aware \& unaware) - in line with previous findings, showing no modulation of the ERN by error awareness

- Differences in Pe not generated in RCZ

- Error related RCZ activity is not sufficient for conscious error awarenes

- Errors may be detected by RCZ without being consciously perceived

Insular Cortex

- Interoceptive awareness (Critchley et al., 2004) and regulation of autonomic responses

- Enhanced awareness of the autonomic reaction to an error or higher autonomic response itself (direction of relationship cannot be revealed by this study)

- Feeling of having committed an error via visceral reactions to the erroneous event

Insula by itself unlikely to generate directly the Pe; but maybe via interactions with other cortical areas involved in generating the $\mathrm{Pe}$

Positive correlation between pre-SMA and post-error slowing after an aware error

- Posterior medial frontal cortex (pMFC) involved in signalling the need for performance adjustments - Post-error slowing more likely when errors are consciously perceived

- Post-error slowing more likely when errors are consciously perceived Increased RCZ activity alone is insufficient to initiate post-error speed-accuracy
on aware errors while RCZ activity is the same for aware and unaware errors)

- Additional processes must co-occur with RCZ activity to enable performance adjustments in $\mathrm{N}+1$

\section{Conclusion}

- RCZ and the anterior, inferior insular cortex around the polus insulae seem both important constituents of the performance monitoring system

RCZ/pFMC activity seems to signal the need for post-error adjustments, it can fulfil this function only when additional conditions - most likely a sufficiently strong general error signal in the cognitive system - are met

- Activity in the anterior inferior insular cortex covaries with error awareness and seems to reflect the awareness of the autonomic response to the error and/or the implementation of this autonomic response itself

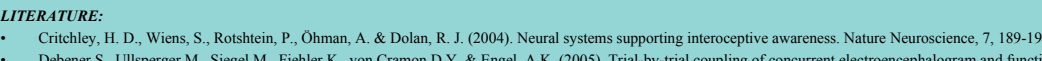

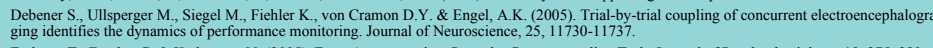

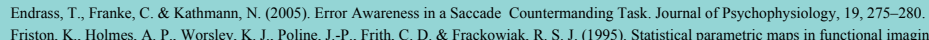

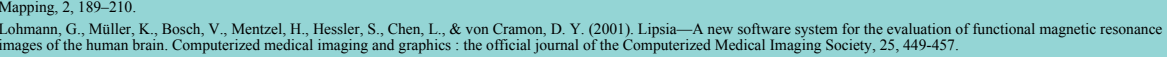

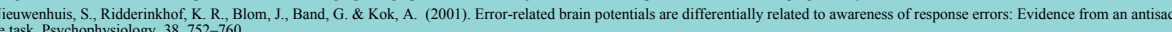
Ridderinhhof, K. R., Ullsperger, M., Crone, E. A. \& Nieuwenhusis, S. (2004). The Role of the Medial Frontal Cortex in Cognitive Control. Science, 306,443 - 447

780.
Figure 5. Reaction times in trial $\mathrm{N}$

Figure 6. Post error reaction times 